\title{
Population Pharmacokinetics and Exposure-Response (Efficacy and Safety/Tolerability) of Empagliflozin in Patients with Type 2 Diabetes
}

Kyle T. Baron · Sreeraj Macha · Uli C. Broedl • Valerie Nock •

Silke Retlich • Matthew Riggs

Received: February 15, 2016/Published online: June 16, 2016

(C) The Author(s) 2016. This article is published with open access at Springerlink.com

\section{ABSTRACT}

Introduction: The aim of the analysis was to characterize the population pharmacokinetics (PKs) and exposure-response (E-R) for efficacy (fasting plasma glucose, glycated hemoglobin) and safety/tolerability [hypoglycemia, genital infections, urinary tract infection (UTI), and volume depletion] of the sodium glucose cotransporter 2 inhibitor, empagliflozin, in patients with type 2 diabetes mellitus. This

Enhanced content To view enhanced content for this article go to http://www.medengine.com/Redeem/ 43D4F0601F78C486.

Electronic supplementary material The online version of this article (doi:10.1007/s13300-016-0174-y) contains supplementary material, which is available to authorized users.

K. T. Baron · M. Riggs

Metrum Research Group, Tariffville, CT, USA

S. Macha

Boehringer Ingelheim Pharmaceuticals, Inc., Ridgefield, CT, USA

U. C. Broedl

Boehringer Ingelheim Pharma GmbH \& Co. KG, Ingelheim, Germany

\section{Nock · S. Retlich $(\bowtie)$}

Boehringer Ingelheim Pharma GmbH \& Co. KG, Birkendorfer Strasse 65, 88397 Biberach, Germany e-mail: silke.retlich@boehringer-ingelheim.com study extends the findings of previous analyses which described the PK and pharmacodynamics (PD) using early clinical studies of up to 12 weeks in duration.

Methods: Population pharmacokinetic and E-R models were developed based on two Phase I, four Phase II, and four Phase III studies.

Results: Variability in empagliflozin exposure was primarily affected by estimated glomerular filtration rate (eGFR) (less than twofold increase in exposure in patients with severe renal impairment). Consistent with its mode of action, the efficacy of empagliflozin was increased with elevated baseline plasma glucose levels and attenuated with decreasing renal function, but was still maintained to nearly half the maximal effect with eGFR as low as $30 \mathrm{~mL} / \mathrm{min} / 1.73 \mathrm{~m}^{2}$. All other investigated covariates, including sex, body mass index, race, and age did not alter the PK or efficacy of empagliflozin to a clinically relevant extent. Compared with placebo, empagliflozin administration was associated with an exposure-independent increase in the incidence of genital infections and no significant change in the risk of UTI, hypoglycemia, or volume depletion. 
Conclusion: Based on the results from the PK and E-R analysis, no dose adjustment is required for empagliflozin in the patient population for which the drug is approved.

Funding: Boehringer Ingelheim.

Keywords: Efficacy;

Exposure-response;

Population pharmacokinetics; Tolerability;

Type 2 diabetes mellitus

\section{INTRODUCTION}

Type 2 diabetes mellitus (T2DM) is a progressive disease, and currently available oral antidiabetic agents, although initially effective, often fail to maintain long-term glycemic control or are associated with side effects, such as hypoglycemia, weight gain, and edema $[1,2]$. Hence, there remains a need for new or alternative therapies that can be used alone or in combination with other antidiabetic agents, and provide sustained improvements in glycemic control without clinically limiting side effects.

A new approach to the management of T2DM involves the reduction of renal glucose reabsorption through inhibition of the sodium glucose cotransporter 2 (SGLT2), found in the brush border of the proximal convoluted tubule of the renal nephron [3]. The inhibition of SGLT2 lowers the renal threshold for glucose reabsorption and increases urinary glucose excretion (UGE) [4-6]. Thereby, SGLT2 inhibition lowers postprandial and fasting plasma glucose (FPG) [7], and reduces glycated hemoglobin (HbA1c) [8]. Empagliflozin is an SGLT2 inhibitor that has been evaluated in Caucasian [9] and Japanese [10] healthy volunteers, and patients with T2DM [7, 11], and demonstrated dose-proportional drug exposure and an increasing UGE with rising empagliflozin doses, up to $10-25 \mathrm{mg}$ [12]. Phase III studies of empagliflozin, administered as monotherapy or add-on to other antidiabetic therapies, have shown improvements in glycemic control, in addition to modest reductions in body weight and blood pressure. Placebo-corrected reductions in HbA1c have been demonstrated both with empagliflozin monotherapy $(-0.7 \%$ for $10 \mathrm{mg}$ and $-0.9 \%$ for $25 \mathrm{mg}$ ) [13], and as add-on therapy $(-0.4 \%$ to $-0.7 \%$ for $10 \mathrm{mg}$ and $-0.5 \%$ to $-0.7 \%$ for $25 \mathrm{mg}$ ) [14-17], in addition to reductions in FPG (monotherapy, $-1.7 \mathrm{mM}$ for $10 \mathrm{mg}$, and $-2.0 \mathrm{mM}$ for $25 \mathrm{mg}$; add-on therapy, -0.9 to $-1.5 \mathrm{mM}$ for $10 \mathrm{mg}$ and -1.2 to $-1.8 \mathrm{mM}$ for $25 \mathrm{mg}$ ) [13-17].

The aim of the present analysis was to characterize the population pharmacokinetics (PK) and exposure-response (E-R) for efficacy and safety/tolerability endpoints of empagliflozin in patients with T2DM. This study extends the findings of previous analyses $[18,19]$, which described the PK and pharmacodynamics (PD) (UGE, FPG, and HbA1c) based on Phase I and II data only. The focus of the present study was to evaluate the impact of covariates, including age, body mass index (BMI), sex, and estimated glomerular filtration rate (eGFR) on the PK and E-R of empagliflozin. The model was used to simulate HbA1c lowering in patient subpopulations of special interest, including elderly patients and individuals with renal impairment.

\section{METHODS}

\section{Population PK Analysis}

\section{Model Development}

A previously developed two-compartment model with a lagged first-order absorption and 
first-order elimination [19] was updated and served as the structural model for the covariate analysis. The effects of the following covariates on the PK of empagliflozin were investigated: age, BMI, sex, race, smoking status, total serum protein, and eGFR using the modification of diet in renal disease formula (MDRD), alanine transaminase (ALT), aspartate transaminase (AST), alkaline phosphatase (AP), and lactate dehydrogenase (LDH). Covariate analysis was conducted using a full covariate modeling approach, testing all of the covariates for their influence on apparent oral clearance (CL/F). The influence of age, sex, race, total protein, and BMI was assessed for the volumes of distribution [apparent central volume of distribution $\left(V_{2} / F\right)$, apparent peripheral volume of distribution $\left.\left(V_{3} / F\right)\right]$, and age, sex, and race were evaluated for influence on the absorption rate constant $\left(k_{\mathrm{a}}\right)$ (Table $\mathrm{S} 1$ in the supplementary material). The absorption lag time was fixed to $0.5 \mathrm{~h}$, and data obtained within the first hour after dosing were excluded from analysis.

The covariates were chosen based on one or a combination of the following: findings from previous analysis $(\mathrm{CL} / F$ : age, BMI, race, total protein, eGFR; $V_{2} / F$ : sex, race, total protein, BMI; $k_{\mathrm{a}}$ : race), scientific interest and relevance to the development programme $\left(V_{2} / F\right.$ : age, race; $k_{\mathrm{a}}$ : sex), physiological/mechanistic plausibility (CL/F: eGFR; $k_{\mathrm{a}}$ : age). Additionally, some covariates were identified during the graphical covariate analysis (CL/F: sex, smoking status). Laboratory tests were included in the full covariate analysis only if a plausible mechanism for their influence on PK variability was known.

The full covariate modeling approach that was implemented is a simplification of a previously described global model approach [20], which emphasizes parameter estimation rather than stepwise hypothesis testing. Predefined covariate parameter relationships were identified based on exploratory graphics, scientific interest, mechanistic plausibility, or prior knowledge, and a full model was constructed with attention to avoiding correlation or co-linearity in predictors. Population typical parameters, including fixed effects parameters (covariate coefficients and structural model parameters) and random effects parameters were estimated using NONMEM $^{\circledR}$ (Version 7.2, ICON Development Solutions, Hanover, MD, USA).

$$
\mathrm{TVP}_{i}=\theta_{n} \cdot \prod_{l}^{m}\left(\frac{\operatorname{cov}_{m i}}{\operatorname{ref}_{m}}\right)^{\theta_{(m+n)}} \cdot \prod_{l}^{p} \theta_{(p+m+n)}^{\operatorname{cov}_{p i}}
$$

where the typical value of a model parameter (TVP) is described as a function of $m$ individual continuous covariates $\left(\operatorname{cov}_{m i}\right)$ and $p$ individual $(0-1)$ categorical covariates $\left(\operatorname{cov}_{p i}\right)$, such that $\theta_{n}$ is an estimated parameter describing the typical PK parameter value for an individual with covariates equal to the reference covariate values $\left(\operatorname{cov}_{m i}=\operatorname{ref}_{m}, \quad \operatorname{cov}_{p i}=0\right) ; \quad \theta_{(m+n)}, \quad$ and $\theta_{(p+m+n)}$ are estimated parameters describing the magnitude of the covariate-parameter relationships.

Patient-level random effects were included for $\mathrm{CL} / F, k_{\mathrm{a}}$, and $V_{3} / F$; these parameters were assumed to be log-normal distributed. Residual unexplained error was modeled with a proportional error model. Further detail is given in Table $\mathrm{S} 1$ in the supplementary material.

Estimates of the covariate effects were classified according to two factors: (1) statistical significance, and (2) magnitude of the effect. A covariate was considered statistically significant if the $95 \%$ bootstrap confidence interval (CI) around the covariate effect estimate excluded the no-effect (null) 
value, otherwise, it was considered non-significant. In line with the bioequivalence criteria, the 0.8-1.25 region was used to rank the magnitude of the covariate effects. If the median normalized covariate effect (i.e., percentage change relative to the reference covariate group) at the extreme covariate levels (as shown in the covariate forest plots) was within this region, the covariate was considered to have only a negligible effect on the PK of empagliflozin.

\section{Model Evaluation}

The precision of the PK model parameter estimates was investigated using a non-parametric bootstrap procedure. In addition, Monte Carlo simulations were performed to evaluate the predictive performance of the population PK model for dose-normalized maximum and minimum concentration $\left(C_{\max }\right.$ and $C_{\min }$, respectively).

\section{Exposure-Response Analysis (Efficacy)}

\section{Model Development}

A population $\mathrm{PK} / \mathrm{PD}$ analysis was conducted using the non-linear mixed-effects modeling software, $\mathrm{NONMEM}^{\circledR}$, to investigate the impact of empagliflozin exposure and selected covariate on FPG and HbA1c. In the population PK/PD model, an increase in empagliflozin exposure was associated with an increase in glucose elimination leading to a reduction in FPG over time (Eq. 1), and thereby, a reduction in HbA1c (Eq. 2). The model structure used in the present study was based on a previously developed PK/PD model [18] and is similar to a model reported for an analysis of multiple treatments for T2DM [21].

$$
\frac{\mathrm{d}\left(\mathrm{FPG}_{i, j}\right)}{\mathrm{d} t}=k_{\mathrm{FPG}_{\mathrm{in}, i}}-k_{\mathrm{FPG}_{\text {out }}} \cdot \mathrm{FPG}_{i, j} \cdot\left(1+\mathrm{STIM}_{i, j}\right)
$$

where $k_{\mathrm{FPG}_{\mathrm{in}, i}}$ is the zero-order production rate constant for FPG, $k_{\mathrm{FPG}_{\text {out }}}$ is the first-order elimination rate constant, and $\operatorname{STIM}_{i, j}$ is the non-linear $\mathrm{E}_{\max }$ expression describing the effect of exposure $\left(\mathrm{AUC}_{\mathrm{ssi}, \mathrm{j}}\right)$ on FPG elimination.

$$
\mathrm{STIM}_{i, j}=\frac{G_{\mathrm{max}_{i}} \cdot \mathrm{AUC}_{\mathrm{ss}_{i, j}}}{\mathrm{AUC}_{50}+\mathrm{AUC}_{\mathrm{ss}_{i, j}}}
$$

where $G_{\text {max }_{i}}$ is the maximal effect for stimulating FPG removal for the $i$ th individual, $\mathrm{AUC}_{50}$ was the $\mathrm{AUC}_{\mathrm{ss}}$ (area under the concentration-time curve at steady-state) that resulted in half the maximal effect, and $\mathrm{AUC}_{\mathrm{ssi}, j}$ was the empagliflozin exposure in the ith patient at the $j$ th collection time affecting the stimulation in FPG removal. A steady-state $\left(\frac{\mathrm{d}\left(\mathrm{FPG}_{i, j}\right)}{\mathrm{d} t}=0\right)$ assumption was made to solve for $k_{\mathrm{FPG}_{\mathrm{in}, i}}$ under initial conditions. This parameterization included the estimation of a baseline FPG (BFPG), as shown in the equations below.

$$
k_{\mathrm{FPG}_{\mathrm{in}, i}}=\mathrm{BFPG}_{i} \cdot k_{\mathrm{FPG}_{\text {out }}}
$$

In turn, changes in FPG over time were modeled to impact HbA1c production, as described by Eq. 2 .

$$
\begin{aligned}
& \frac{\mathrm{d}\left(\mathrm{HbA}_{1} \mathrm{c}_{i, j}\right)}{\mathrm{d} t}=k_{\mathrm{HbAlc}_{\mathrm{in}, i}} \times \mathrm{FPG}_{i, j}-k_{\mathrm{HbA1} \mathrm{c}_{\mathrm{out}, i}} \\
& \cdot \operatorname{HbA} \mathrm{c}_{i, j} \cdot\left(1-\frac{\mathrm{HbA} 1 \mathrm{c}_{\text {limit }}}{\mathrm{HbA}_{\mathrm{c}} \mathrm{c}_{i, j}}\right)
\end{aligned}
$$

where $k_{\mathrm{HbAlc} \text { in, } i}$ was the first-order production rate constant of HbA1c, HbA1c $c_{i, j}$ was the HbA1c value for the $i$ th patient at the $j$ th collection time, $k_{\mathrm{HbAlc}_{\text {out }, i}}$ was the first-order elimination rate constant, and $\mathrm{HbA} 1 \mathrm{c}_{\text {limit }}$ was the boundary condition.

Based on this structural model, a covariate analysis was performed. The covariates of sex, race, BMI, eGFR, BFPG, duration of T2DM, and concomitant antidiabetic therapies [metformin, 
sulfonylurea (SU), pioglitazone] were investigated as predictors for the extent of FPG reduction $\left(G_{\max }\right)$. Covariates for $k_{\mathrm{HbAlc} \text { out }, i}$ were concomitant metformin, duration of T2DM, eGFR, BMI, Asian race, and sex. For the PK/PD covariate analysis, covariates were included based on the knowledge from previous analysis (BFPG: observed FPG baseline;

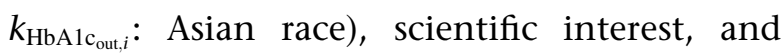
relevance to development programme (BFPG: duration of diabetes, sex; $G_{\max }$ : demographics;

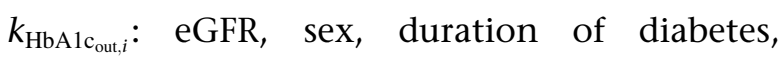
concomitant metformin), mechanistic/ physiological plausibility (BFPG: age, BMI, concomitant medication, number/types of previous treatments; $G_{\max }$ : eGFR, observed BFPG; concomitant antidiabetic medication, duration of diabetes), and graphical analysis (BFPG: eGFR; $k_{\mathrm{HbAlc} \text { out }, i}$ : demographics).

\section{Model Evaluation}

The model development was performed in a stepwise manner. First, an initial model was developed excluding studies 6 and 10 (as a result of data availability). For this initial model, an external simulation check was performed, where the HbA1c reductions in study 6 were predicted and compared with the original data from this trial. Study 6 was selected for external evaluation as its patient population was similar to the one used for model development. Finally, the model was updated using all studies, including studies 6 and 10. The precision of the final PK/PD model parameter estimates was investigated using a non-parametric bootstrap procedure. In addition, Monte Carlo simulations were performed to evaluate the predictive performance of the final population PK/PD model for change in HbA1c.

\section{Simulations}

The simulations performed for the final predictive check provided guidance on the typical magnitude and time-course of HbA1c response. Additional simulations $(n=1000$ studies, including inter-individual + residual variability) were performed to assess $\mathrm{HbA1c}$ lowering after 24 weeks of treatment in subpopulations of special interest, i.e., patients with renal impairment (study 10) and patients of advanced age (final PK/PD dataset, patients aged 75-85 years). The covariate values (such as BMI, eGFR, and age) that were associated with the patients in each subpopulation were accounted for in the simulations. For the purpose of the simulations, it was assumed that each patient received either $10 \mathrm{mg}$ (scenario 1) or $25 \mathrm{mg}$ (scenario 2) empagliflozin once daily. Only patients on active treatment in the original study were considered because the calculation of the individual $\mathrm{AUC}_{\mathrm{ss}}$ values required an estimate of $\mathrm{CL} / F_{\mathrm{i}}$.

\section{Exposure-Response Analysis (Safety)}

\section{Model Development}

The safety/tolerability endpoints investigated in the E-R analysis were confirmed hypoglycemic adverse events (AEs) (plasma glucose $<3.9 \mathrm{mM}$ and/or assistance required), events consistent with urinary tract infection (UTI; using a prospectively defined search of 73 preferred terms), events consistent with genital infection (using a prospectively defined search of 89 preferred terms), and events consistent with volume depletion (all on-treatment AEs, using eight preferred terms).

The safety/tolerability endpoints considered in the E-R analysis were included as dichotomous endpoints (i.e., participant-reported AEs: any AE during study, single yes/no datapoint), and were 
analyzed using a logistic regression model $[19,22]$. Odds ratios (ORs) for an event on empagliflozin treatment were calculated and independent variables were incorporated into the model via the logit function. The covariates evaluated included age, Asian race, renal function stage, sex, concomitant oral (metformin and SU) and parenteral (insulin) antidiabetic agents, and empagliflozin exposure. Covariate effects were included using the full covariate modeling approach $[19,22]$. If covariates were included, they were entered additively using the formula below.

$$
\begin{aligned}
\lambda_{i}= & \beta_{0}+\beta_{n}^{\text {Categorical }} \\
& +\beta_{n+1}\left(\text { Continuous }- \text { Continuous }_{\text {ref }}\right) \\
& +\beta_{n+2}\left(\frac{\mathrm{AUC}_{\mathrm{SS}, i}}{\mathrm{AUC} \mathrm{SS}_{\mathrm{ref}}}\right)
\end{aligned}
$$

where the logit function, $\lambda_{i}=\log \left(\frac{p_{i}}{1-p_{i}}\right)$ and $\mathrm{p}_{i}$ is the AE probability for the ith patient, Continuous $_{\text {ref }}$ is the reference value for a continuous covariate, $\mathrm{AUC}_{\mathrm{SS}, \text { ref }}$ is the reference empagliflozin AUC at steady-state, and $\mathrm{AUC}_{\mathrm{Ss}, i}$ is the AUC at steady-state in the ith patient. Interaction terms were included in the model for hypoglycemic AEs when a patient was on insulin + metformin or insulin + metformin + SU. Logistic regression models were fitted in $\mathrm{R}$ version 2.12.2 [23] using the $\mathrm{glm}$ function for binomial likelihood and logit link function.

It should be noted that non-exposure related covariates were only included for the purpose of adjusting the event rates to allow for proper assessment of the effect of exposure on the different $\mathrm{AE}$ rates. These non-exposure related covariates could, therefore, be regarded solely as adjustment factors and not as points for inference. Selection of adjustment covariates was, therefore, data driven and dependent on the endpoint that was analyzed.
Note also that this article is based on the analysis of data from previously conducted studies, and does not involve any new studies of human or animal subjects performed by any of the authors.

\section{RESULTS}

The population PK analysis was based on 12,503 empagliflozin plasma concentrations from two Phase I, four Phase II, and four Phase III studies (2761 patients on active empagliflozin) (Table 1) [7, 8, 11-13, 15, 17, 24]. Doses of orally administered empagliflozin ranged from 1 to $100 \mathrm{mg}$, with 1129 patients (40.9\%) receiving $10 \mathrm{mg}$ and 1269 patients (46.0\%) receiving $25 \mathrm{mg}$ empagliflozin once daily. Patients receiving placebo were not included in the PK analysis.

\section{Population PK Analysis}

The PK of empagliflozin was well described by a two-compartment model with first-order absorption (Figs. S1 and S2 in the supplementary material). Model parameters were estimated with reliable precision and inclusion of covariate effects within this model described a portion of the inter-individual variability. Typical population PK parameters (95\% CI, based on non-parametric bootstrap) given the reference covariates (50-year-old, non-smoking male, non-Asian race; BMI, $25 \mathrm{~kg} / \mathrm{m}^{2}$; eGFR, $100 \mathrm{~mL} / \mathrm{min} / 1.73 \mathrm{~m}^{2}$; total protein, $70 \mathrm{~g} / \mathrm{dL}$; ALT, $20 \mathrm{U} / \mathrm{L}$; AST, $20 \mathrm{U} / \mathrm{L}$; AP, $70 \mathrm{U} / \mathrm{L}$; and LDH, $160 \mathrm{U} / \mathrm{L}$ ) were: $\mathrm{CL} / F, 10.6 \mathrm{~L} / \mathrm{h}$ $(10.1, \quad 11.1) ; \quad V_{2} / F, \quad 3.14 \mathrm{~L} \quad(0.00128,4.03)$; apparent intercompartmental clearance $(Q / F)$, $6.34 \mathrm{~L} / \mathrm{h}(5.72,6.91) ; V_{3} / F, 70.6 \mathrm{~L}(64.4,76.6)$; and first-order $k_{\mathrm{a}}, 0.196 \mathrm{~L} / \mathrm{h}(0.185,0.208)$. 


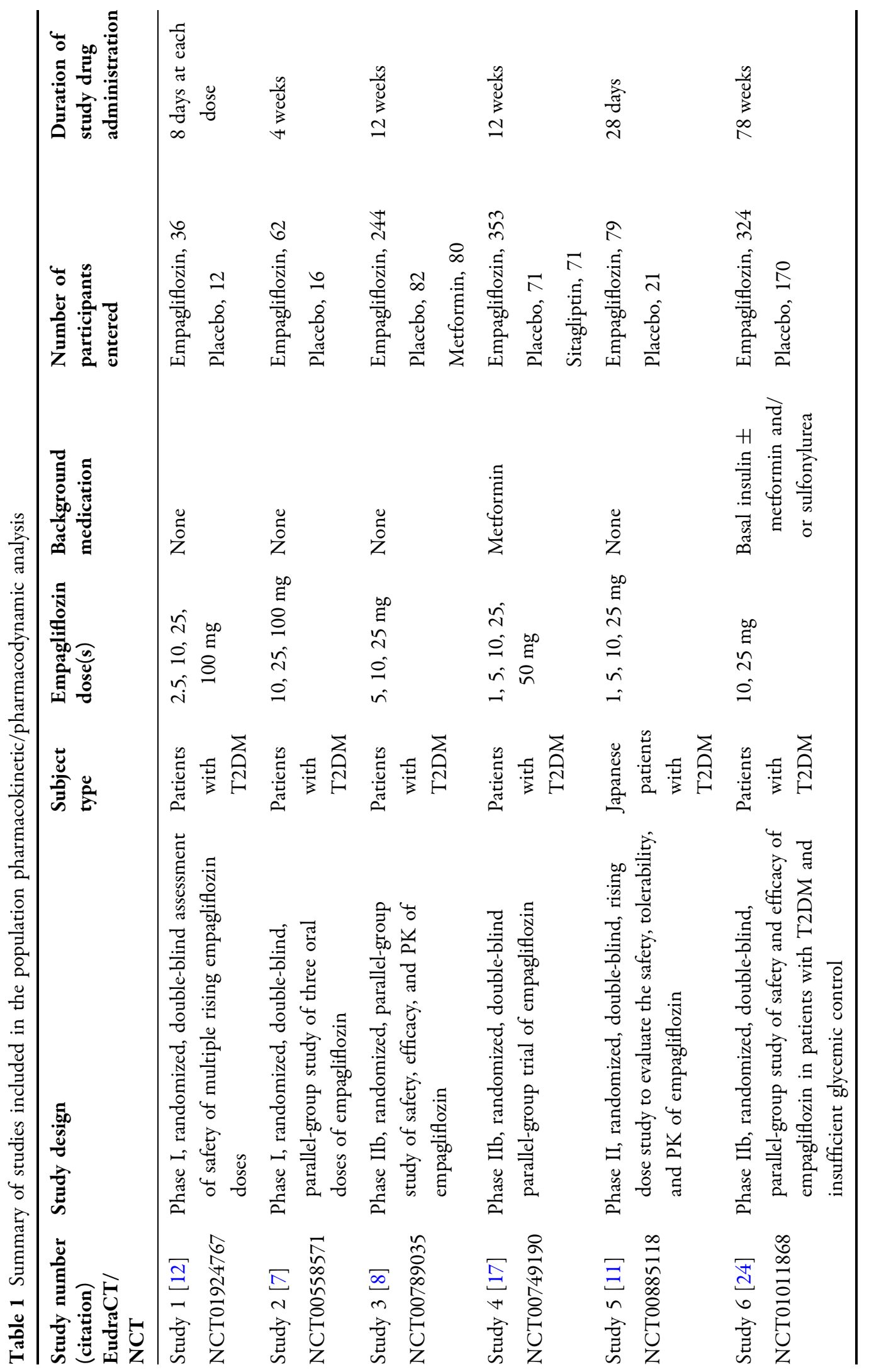




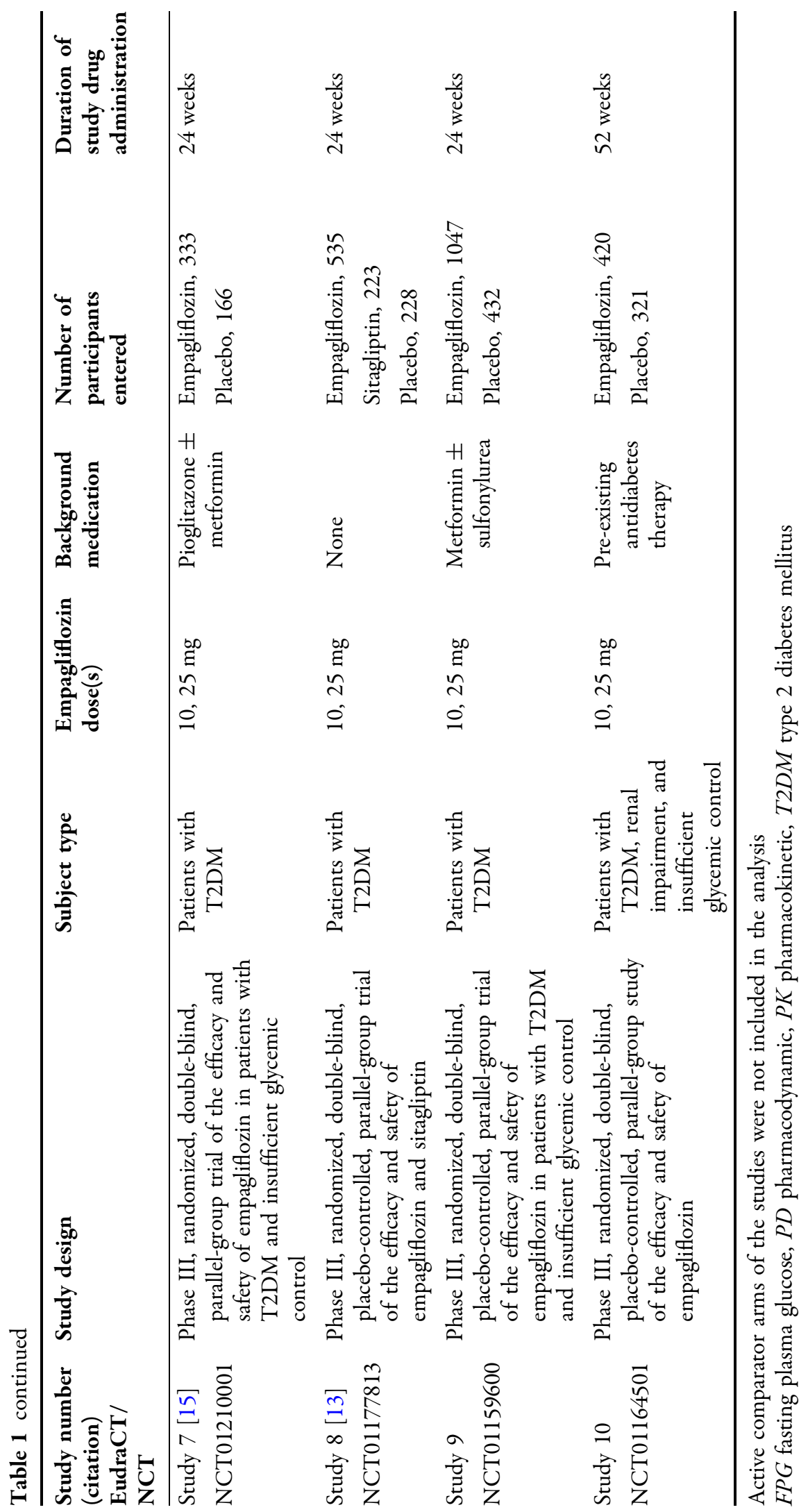


a

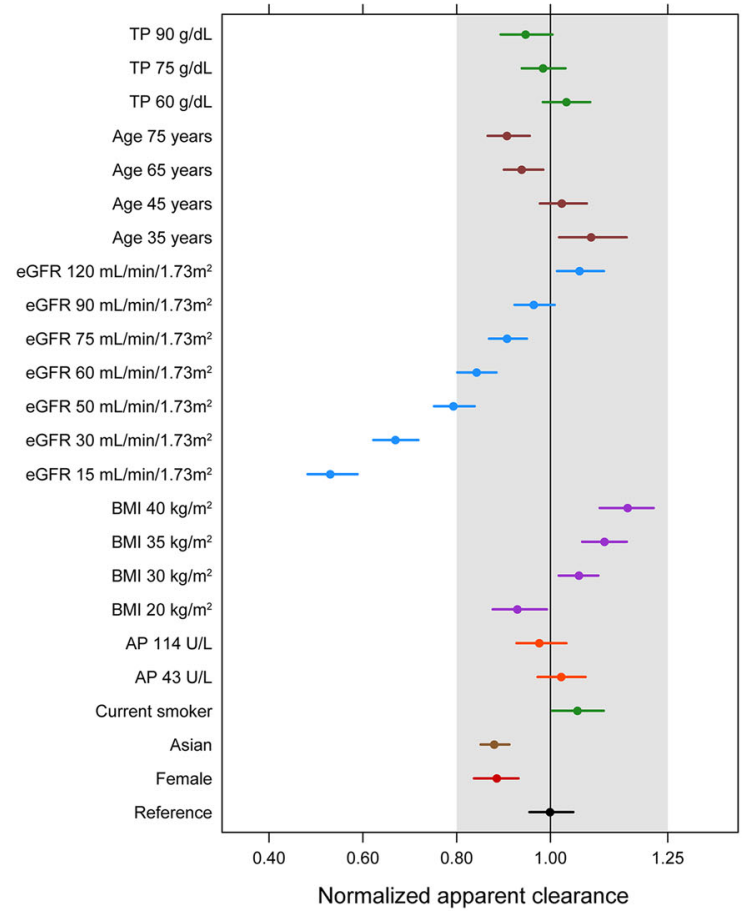

Fig. 1 Covariate effects on $\mathbf{a} \mathrm{CL} / \mathrm{F}$ and $\mathbf{b}$ relative empagliflozin exposure $\left(\mathrm{AUC}_{\mathrm{ss}} /\right.$ reference $\left.\mathrm{AUC}_{\mathrm{ss}}\right)$. Reference group: male, non-Asian, non-smoker, total protein $70 \mathrm{~g} / \mathrm{dL}$, eGFR $100 \mathrm{~mL} / \mathrm{min} / 1.73 \mathrm{~m}^{2}$, ALT $20 \mathrm{U} / \mathrm{L}$, AST $20 \mathrm{U} / \mathrm{L}, \mathrm{AP} 70 \mathrm{U} / \mathrm{L}, \mathrm{LDH} 160 \mathrm{U} / \mathrm{L}, \mathrm{BMI} 25 \mathrm{~kg} / \mathrm{m}^{2}$, age 50 years. $A L T$ alanine transaminase, $A P$ alkaline

Figure 1 shows the estimated covariate effects on $\mathrm{CL} / F$ (Fig. 1a) and relative empagliflozin exposure (Fig. 1b) from the population PK model, and demonstrates a prominent correlation between decreasing eGFR and $\mathrm{CL} / F$. Typical $\mathrm{AUC}_{\mathrm{ss}}$ values were increased in the presence of renal impairment: $\mathrm{AUC}_{\mathrm{ss}}$ increased by $18.5 \%(95 \%$ CI 13.0, 24.8), 49.2\% (39.2, 60.6), and $88.1 \%$ $(69.9,107.0)$ in patients with eGFR of 60,30 , and $\quad 15 \mathrm{~mL} / \mathrm{min} / 1.73 \mathrm{~m}^{2}$, respectively, compared with a reference patient with an eGFR of $100 \mathrm{~mL} / \mathrm{min} / 1.73 \mathrm{~m}^{2}$. However, typical $\mathrm{AUC}_{\mathrm{ss}}$ values were increased less than twofold in the presence of renal impairment $\left(88.1 \%\right.$ for an eGFR of $\left.15 \mathrm{~mL} / \mathrm{min} / 1.73 \mathrm{~m}^{2}\right)$.

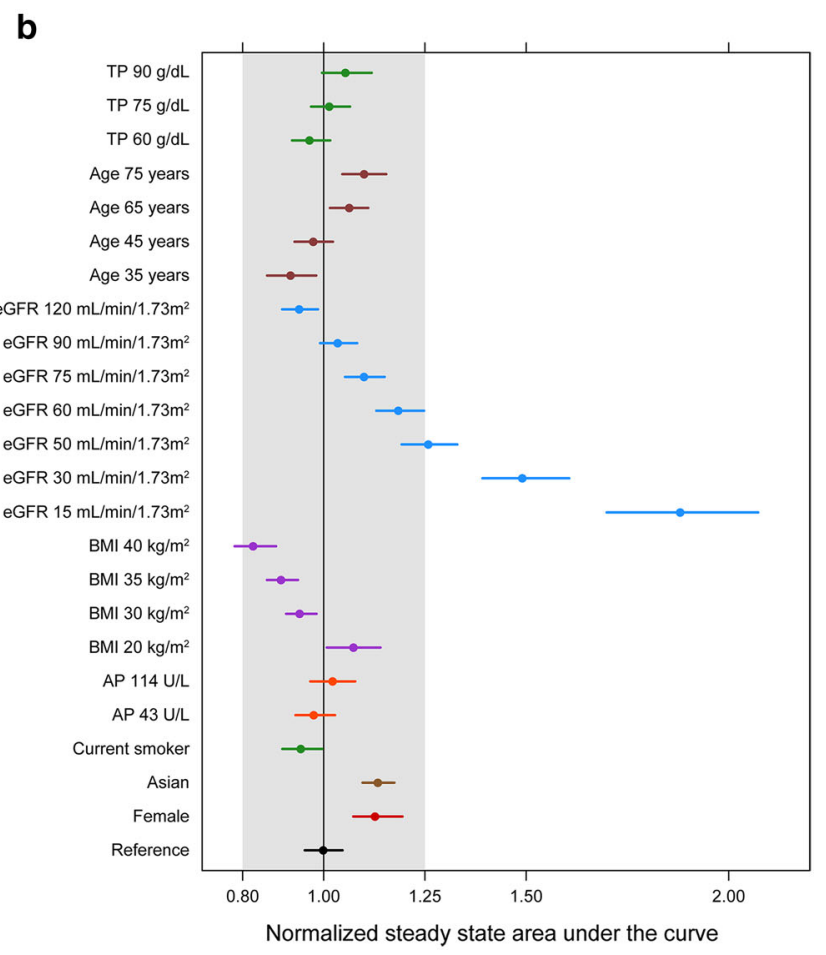

phosphatase, $A S T$ aspartate transaminase, $A U C$ area under the concentration-time curve, $B M I$ body mass index, $e G F R$ estimated glomerular filtration rate, $L D H$ lactate dehydrogenase, $P D$ pharmacodynamic, $P K$ pharmacokinetic, $T P$ total protein

The analysis also showed statistically significant relationships between BMI, total protein, age, female sex, current smoking status, Asian race, and AP and CL/F (95\% CIs for these estimates did not include the null value). However, the magnitudes of these covariate effects were minor, with differences within these covariate groups between 0.8 and 1.25 of the normalized $\mathrm{CL} / F$ or $\mathrm{AUC}_{\mathrm{ss}}$ at the extreme covariate levels (Fig. 1). The other covariate effects (ALT, AST, LDH, and previous smoking history) were non-significant. Estimated covariate effects on parameters not affecting $\mathrm{AUC}_{\mathrm{ss}}\left(V_{2} / \mathrm{F}, V_{3} /\right.$ $F$, and $k_{\mathrm{a}}$ ) are included in Table $\mathrm{S} 1$ in the supplementary material. 
Table 2 Summary of continuous covariates

\begin{tabular}{llll}
\hline Covariate & PK/PD dataset & $\begin{array}{l}\text { Subdataset: elderly } \\
(75-85 \text { years })\end{array}$ & $\begin{array}{l}\text { Subdataset: renal } \\
\text { impairment }(\text { study 10) }\end{array}$ \\
\hline Age, years (SD) & $58.0(36.0,76.0)$ & $77.0(75.0,84.7)$ & $63.0(46.9,81.4)$ \\
eGFR, $\mathrm{mL} / \mathrm{min} / 1.73 \mathrm{~m}^{2}$ & $81.8(33.4,128)$ & $61.5(20.5,93.0)$ & $60.1(22.1,86.8)$ \\
$\mathrm{BMI}, \mathrm{kg} / \mathrm{m}^{2}$ & $29.1(21.0,42.4)$ & $29.4(21.8,38.3)$ & $30.1(20.8,42.3)$ \\
Total protein, g/dL & $72.0(64.0,82.0)$ & $71.0(63.0,79.7)$ & $71.0(62.0,81.0)$ \\
AST, U/L & $21.0(12.0,52.0)$ & $21.0(14.0,52.7)$ & $21.0(12.0,47.0)$ \\
ALT, U/L & $25.0(10.6,75.0)$ & $21.5(11.0,48.0)$ & $23.0(10.3,67.0)$ \\
AP, U/L & $73.0(41.0,129)$ & $76.5(41.0,147)$ & $72.0(42.3,134)$ \\
LDH, U/L & $162(114,247)$ & $170(123,258)$ & $170(114,263)$ \\
Baseline FPG, mM & $8.38(4.83,13.6)$ & $8.35(4.57,14.2)$ & $7.99(4.52,11.4)$ \\
Baseline HbA1c, \% & $7.90(6.70,9.80)$ & $7.85(6.73,9.77)$ & $7.90(6.73,9.50)$ \\
\hline
\end{tabular}

Data are median (2.5th and 97.5th percentile)

Number of patients in PK/PD dataset: 4065

Number of patients in elderly simulation dataset: 94 (reflects subdataset of PK/PD dataset, filtered for patients aged $75-85$ years who were receiving empagliflozin, 10 and $25 \mathrm{mg}$ )

Number of patients in renal impairment simulation dataset: 253 (patients in study 10 on empagliflozin, 10 and $25 \mathrm{mg}$ ) Influence of eGFR on model parameters was estimated based on final PK/PD dataset containing more patients with renal impairment than those from study 10

$A L T$ alanine transaminase, $A P$ alkaline phosphatase, $A S T$ aspartate transaminase, $B F P G$ baseline fasting plasma glucose, $B M I$ body mass index, $e G F R$ estimated glomerular filtration rate, $F P G$ fasting plasma glucose, HbAlc glycated hemoglobin, $L D H$ lactate dehydrogenase, $P D$ pharmacodynamic, $P K$ pharmacokinetic, $S D$ standard deviation

\section{Exposure-Response Analysis (Efficacy)}

Fasting plasma glucose measurements from the same studies were used to develop the E-R model for FPG (4289 patients), including patients receiving empagliflozin or placebo. The population PK/PD dataset for the FPG/ HbA1c model comprised HbA1c measurements from studies of $\geq 12$ weeks' duration, i.e., three Phase II trials and four Phase III trials (4065 patients, including placebo arms, with 25,361 FPG values and 22,012 HbA1c assessments).

Two indirect-response models were implemented to describe the impact of empagliflozin exposure on the efficacy endpoints FPG and HbA1c. A rise in empagliflozin exposure was associated with increased glucose elimination, which led to a reduction in FPG, and thereby, a decrease in HbA1c. For an initial model, developed without studies 6 and 10, an external simulation check was performed showing that the model adequately predicted the HbA1c reductions in study 6 (Fig. S3 in the supplementary material). The performance of the final PK/PD models comprising all data was evaluated using goodness of fit plots and visual predictive checks, which indicated that the model adequately described the efficacy data (Figs. S4-S7 in the supplementary material).

The continuous and categorical covariates for the PK/PD datasets are summarized in Table 2 and Table S2 in the supplementary material. For the population PK/PD efficacy 


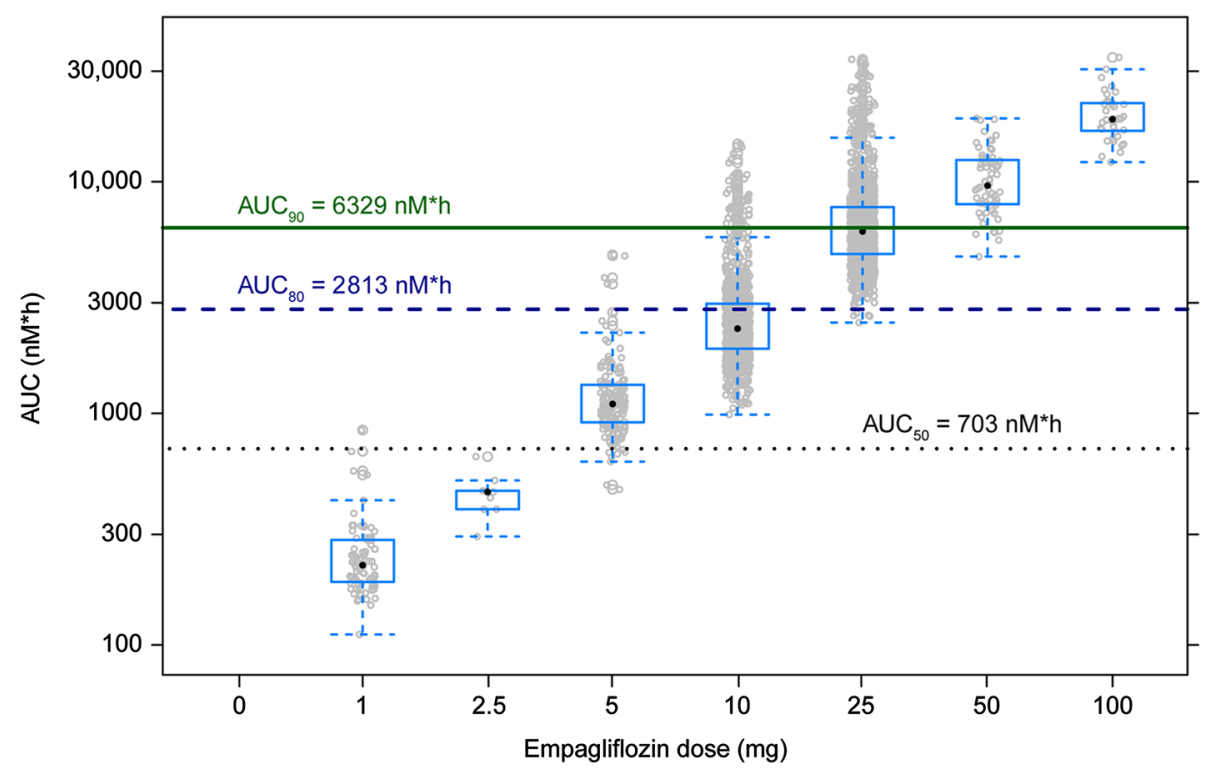

Fig. 2 Individually estimated AUC values by empagliflozin dose compared with the estimated $\mathrm{AUC}_{50}, \mathrm{AUC}_{80}$, and $\mathrm{AUC}_{90} . A U C$ area under the concentration-time curve

analysis, median BFPG was $8 \mathrm{mM}(144 \mathrm{mg} / \mathrm{dL})$ for studies 1, 2, and 6-10, and $9 \mathrm{mM}(162 \mathrm{mg} /$ dL) for studies 3-5. Evaluated covariates (age, BMI, sex, race, eGFR, concomitant antidiabetic therapy, and duration of T2DM) had only minimal impact $(<7 \%)$ on BFPG. Median $\mathrm{AUC}_{50}$ was $704 \mathrm{nM}^{\star} \mathrm{h}$, corresponding to a median empagliflozin exposure of around $3 \mathrm{mg}$ from a once daily dose (Table S3 in the supplementary material, and Fig. 2).

The maximum reduction $\left(G_{\max }\right)$ in FPG with empagliflozin therapy was estimated to be $22 \%$. $G_{\text {max }}$ was mainly affected by BFPG and eGFR; $G_{\max }$ was estimated to increase with increasing BFPG and decrease with decreasing eGFR. Significant but small effects on $G_{\max }$ were observed with metformin + SU co-treatment, sex, BMI, and age. Other covariates (metformin or pioglitazone co-treatment, duration of T2DM, and Asian race) had no significant effect on $G_{\max }$ of FPG.

Glycated hemoglobin half-life (calculated from $\left.k_{\mathrm{HbA1c}, \text { out }}\right)$ was approximately 2.6 weeks
(95\% CI 1.7, 3.9), indicating that maximum changes in HbA1c are reached by around 12 weeks ( $>3$ half-lives) of empagliflozin therapy, and was almost entirely achieved by 24 weeks ( $>6$ half-lives). Covariate effects relating to HbA1c (estimated using $k_{\mathrm{HbA1c}, \mathrm{out}}$ ) were generally non-significant (i.e., the CIs contained the null value), although were not precisely estimated with the exception of metformin co-medication, which resulted in an increase in $k_{\mathrm{HbA1c} \text {,out, }}$ resulting in a lower $\mathrm{HbA1c}$ baseline for patients pre-treated with metformin.

Based on parameter estimates for the final HbA1c E-R model, targets of $80 \%$ and $90 \%$ were obtained for the maximal response for FPG and HbA1c after 24 weeks of treatment with once daily empagliflozin doses of approximately 10 and $25 \mathrm{mg}$, respectively (Table S4 in the supplementary material).

Simulations were performed to illustrate the impact of the investigated covariates on change in HbA1c from baseline after 24 weeks of treatment with empagliflozin $25 \mathrm{mg}$ for each 
of the single investigated covariates. Following the covariate effects on $G_{\max }, \mathrm{HbA1c}$ lowering was mainly influenced by BFPG and eGFR with a median change from baseline $\mathrm{HbA1c}$ of $-0.59 \%$ (95\% CI $-0.65,-0.53)$ for patients with an eGFR of $60 \mathrm{~mL} / \mathrm{min} / 1.73 \mathrm{~m}^{2}$, and $-0.89 \%(-0.98,-0.82)$ for patients with a BFPG of $9 \mathrm{mM}$, compared with a median change of $-0.71 \% \quad(-0.79,-0.65)$ for a reference patient (eGFR, $100 \mathrm{~mL} / \mathrm{min} / 1.73 \mathrm{~m}^{2}$; BFPG, 8 mM; Fig. 3).

To investigate the influence of eGFR and age on HbA1c lowering, deterministic simulations were performed to illustrate the E-R after 24 weeks of treatment (Fig. S8 in the supplementary

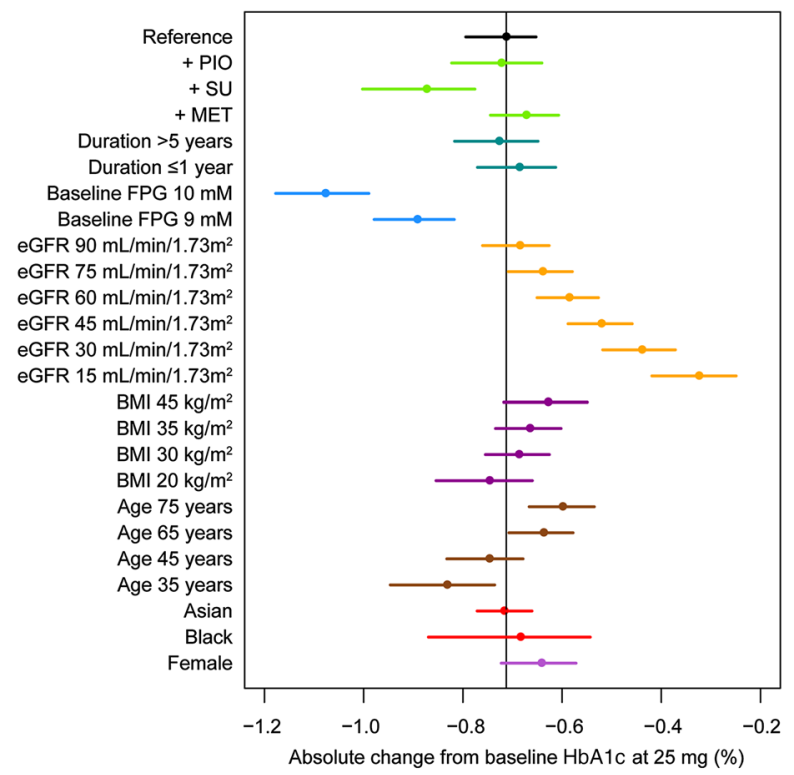

Fig. 3 Covariate effects on HbAlc lowering after 24 weeks of treatment with empagliflozin $25 \mathrm{mg}$. Reference: male; non-black, non-Asian; age 50 years; eGFR $100 \mathrm{~mL} / \mathrm{min} / 1.73 \mathrm{~m}^{2}$; BMI $25 \mathrm{~kg} / \mathrm{m}^{2}$; BFPG $8 \mathrm{mM}$; duration of diabetes 1.5 years with no concomitant antidiabetic therapy. Points represent the median, horizontal lines the $95 \% \mathrm{CI}$ of the covariate effect. CIs were determined from 1000 simulations taking parameter uncertainty into account. $B F P G$ baseline fasting plasma glucose, $B M I$ body mass index, $C I$ confidence interval, eGFR estimated glomerular filtration rate, $F P G$ fasting plasma glucose, $H b A 1 c$ glycated hemoglobin, MET metformin, $P I O$ pioglitazone, $S U$ sulfonylurea material). As these simulations focus only on the influence of age and eGFR but not the combined effect of other covariates, stochastic simulations in the relevant subpopulations [i.e., patients with renal impairment and elderly patients (75-85 years)] were performed to characterize the effect of empagliflozin under more realistic conditions (Table 3).

\section{Exposure-Response Analysis (Safety)}

In total, 4065 patients were evaluable in the safety/tolerability dataset (2584 on active empagliflozin therapy and 1481 on placebo). Overall safety/tolerability event rates were: $11.5 \%(n=466)$ for confirmed hypoglycemic AEs, $8.09 \%(n=329)$ for events consistent with UTI, $2.85 \%(n=116)$ for events consistent with genital infection, and $0.839 \%(n=34$ events reported from a dataset of 4054 patients) for events consistent with volume depletion. The impact of empagliflozin exposure on the tolerability endpoints was adequately described by logistic regression models (Fig. S9 in the supplementary material). The main covariate influences accounted for in the E-R analyses were concomitant insulin therapy for confirmed hypoglycemic events [OR 48.7 (95\% CI 26.5, 89.5)]; female sex for UTI [OR 6.14 $(4.60,8.19)]$, and genital infection [OR 2.28 $(1.55,3.35)] ;$ and renal impairment [eGFR $60-90 \mathrm{~mL} / \mathrm{min} / 1.73 \mathrm{~m}^{2}$; OR $\left.1.75(0.617,4.96)\right]$; eGFR $<60 \mathrm{~mL} / \mathrm{min} / 1.73 \mathrm{~m}^{2}$; OR $2.78 \quad(0.829$, 9.34), and insulin therapy [OR, 2.60 (1.21, 5.61)] for volume depletion. Compared with placebo-treated patients, empagliflozin therapy was associated with an increased incidence of genital infection [OR $5.08(2.77,9.34)]$ but no significant change in the risk of UTI [OR 0.941 (0.687, 1.29)], hypoglycemia [OR 1.11 (0.811, 1.52)], or volume depletion [OR 1.44 (0.517, 4.01)]. For patients receiving empagliflozin 
Table 3 Median change from baseline in glycated hemoglobin after 24 weeks of treatment in subpopulations of elderly patients and patients included in the renal impairment study (study 10)

\begin{tabular}{|c|c|c|c|c|}
\hline \multirow[t]{2}{*}{ Patient population } & \multicolumn{2}{|l|}{$10 \mathrm{mg}$} & \multicolumn{2}{|l|}{$25 \mathrm{mg}$} \\
\hline & Observed & Predicted & Observed & Predicted \\
\hline Elderly ( $75-85$ years) & $-0.65(-2.44,0.40)$ & $-0.48(-0.29,-0.65)$ & $-0.50(-2.03,0.60)$ & $-0.53(-0.36,-0.71)$ \\
\hline $\begin{array}{l}\text { eGFR } 60-90 \mathrm{~mL} / \mathrm{min} / \\
1.73 \mathrm{~m}^{2}\end{array}$ & $-0.40(-1.70,0.76)$ & $-0.53(-0.67,-0.37)$ & $-0.70(-1.64,0.90)$ & $-0.59(-0.74,-0.44)$ \\
\hline $\begin{array}{l}\text { eGFR } 30-60 \mathrm{~mL} / \mathrm{min} / \\
1.73 \mathrm{~m}^{2}\end{array}$ & $-0.90(-1.81,0.12)^{\mathrm{a}}$ & $-0.45(-0.60,-0.29)$ & $-0.40(-2.00,1.25)$ & $-0.49(-0.65,-0.33)$ \\
\hline $\begin{array}{l}\text { eGFR } 45-60 \mathrm{~mL} / \mathrm{min} / \\
1.73 \mathrm{~m}^{2}\end{array}$ & $-0.90(-1.81,0.12)^{\mathrm{a}}$ & $-0.46(-0.69,-0.26)$ & $-0.40(-1.97,0.88)$ & $-0.51(-0.75,-0.30)$ \\
\hline $\begin{array}{l}\text { eGFR } 30-45 \mathrm{~mL} / \mathrm{min} / \\
1.73 \mathrm{~m}^{2}\end{array}$ & - & $-0.42(-0.64,-0.19)$ & $-0.30(-1.83,1.43)$ & $-0.46(-0.69,-0.23)$ \\
\hline
\end{tabular}

eGFR estimated glomerular filtration rate

The elderly subpopulations included all patients aged $75-85$ years $(n=94$, with their associated covariate values) and patients with renal impairment (from study $10, n=253$, with their associated covariates)

For observations, median, 2.5th, and 97.5th percentiles are shown; for simulations, the median and its $95 \% \mathrm{CI}$ is shown Metrics are given for patients having a baseline and a 24-week glycated hemoglobin (HbAlc) measurement only

a Based on observations from five patients who received the 10-mg dose. In study 10, the 10-mg dose was not evaluated in patients with moderate renal impairment. The five patients who showed mild renal impairment (eGFR $>60 \mathrm{~mL} / \mathrm{min} /$ $1.73 \mathrm{~m}^{2}$ ) at screening were assigned to 10-mg empagliflozin, and subsequently, had eGFR values between 49 and $58 \mathrm{~mL} /$ $\min / 1.73 \mathrm{~m}^{2}$ at the baseline visit just prior to treatment initiation. These patients were therefore categorized as having moderate renal impairment, but continued on the randomized 10-mg dose. As simulations are summarized as the change from baseline (defined as start of treatment) HbAlc values, stratification of the patients with respect to the baseline renal impairment category was completed, and hence, five patients categorized as having moderate renal impairment were treated with 10-mg empagliflozin

treatment, increases in empagliflozin $\mathrm{AUC}_{\mathrm{ssi}}$ [OR $(95 \%$ CI) for AUC normalized to $3500 \mathrm{nM}^{*} \mathrm{~h}$ increase in $\left.\mathrm{AUC}_{\mathrm{ss}}\right]$ produced no significant increase in incidence rates of confirmed hypoglycemic AEs [0.988 (0.863, 1.13)], events consistent with volume depletion $[0.770 \quad(0.454,1.30)]$, events consistent with UTI $[1.06(0.935,1.20)]$, or events consistent with genital infection [0.744 $(0.574,0.965)]$.

\section{DISCUSSION}

The population PK and E-R for the efficacy and safety/tolerability of empagliflozin was investigated in patients with T2DM. The aims of the population PK analysis were to describe the PK of empagliflozin in patients with T2DM and to quantify the effects of covariates. The PK of empagliflozin was well described by a two-compartment model with first-order absorption. The covariate analysis indicated that no dose adjustment is necessary for the evaluated covariates. Variability in the $\mathrm{CL} / F$, and hence, $\mathrm{AUC}_{\mathrm{ss}}$ of empagliflozin was statistically significantly affected by eGFR, BMI, total protein, age, female sex, current smoking, and Asian race, but their clinical impact on empagliflozin exposure was minor (i.e., 80-125\%). The only statistically significant 
effect that reached beyond $125 \%$ was the impact of eGFR on $\mathrm{CL} / \mathrm{F}$, and hence, $\mathrm{AUC}_{\mathrm{ss}}$ values. $\mathrm{CL} / F$ values were reduced with declining eGFR with an expected $46.8 \%$ (95\% CI 51.8, 41.1) reduction in $\mathrm{CL} / F$ and $88.1 \%$ (95\% CI $69.9,107)$ increase in $\mathrm{AUC}_{\mathrm{ss}}$ for patients with eGFR of $15 \mathrm{~mL} / \mathrm{min} / 1.73 \mathrm{~m}^{2}$ compared with a reference patient with eGFR of $100 \mathrm{~mL} / \mathrm{min} /$ $1.73 \mathrm{~m}^{2}$. For eGFR $\geq 60 \mathrm{~mL} / \mathrm{min} / 1.73 \mathrm{~m}^{2}$, the mean $\mathrm{AUC}_{\mathrm{ss}}$ was not increased by more than $20 \%$, compared with the reference eGFR of $100 \mathrm{~mL} / \mathrm{min} / 1.73 \mathrm{~m}^{2}$. This finding is in line with the results of a study of Japanese patients with T2DM and renal impairment, as well as a study in subjects with renal impairment, which demonstrated an increase in exposure for patients with renal impairment of less than twofold $[25,26]$. Thus, a dose reduction on the basis of renal function would not be required from a PK perspective.

The E-R analyses for efficacy indicated that the empagliflozin doses of 10 and $25 \mathrm{mg}$ were near the plateau of the maximal achievable HbA1c-lowering effect. The main determinants of the efficacy of empagliflozin therapy were BFPG levels and renal function. The maximal achievable effect was estimated using the $G_{\max }$ parameter, and was shown to increase with increased BFPG and decrease with declining renal function. These observations are consistent with the physiology of glucose excretion, where an increased amount of glucose available for renal filtration will lead to an increase in UGE resulting from SGLT2 inhibition. In the presence of renal impairment, decreased glomerular filtration would be expected to result in a reduction in UGE, a reduced FPG response, and subsequently, a lesser reduction in HbA1c with empagliflozin therapy, compared with individuals with normal renal function [27]. Nonetheless, even with eGFR values as low as $30 \mathrm{~mL} / \mathrm{min} / 1.73 \mathrm{~m}^{2}$, the efficacy of empagliflozin was maintained to nearly half-maximal effect on FPG. Simulations were performed to better characterize the impact of these considerations on $\mathrm{HbA} 1 \mathrm{c}$ lowering. As shown in Fig. 3, the simulations showed a median change from baseline HbA1c of $-0.58 \%$ (95\% CI $-0.65,-0.52)$ for patients with an eGFR of $60 \mathrm{~mL} / \mathrm{min} / 1.73 \mathrm{~m}^{2}$, and $-0.89 \%$ (95\% CI $-0.98,-0.82)$ for patients with a BFPG of $9 \mathrm{mM}$. The analysis also demonstrated a reduced effect on HbA1c lowering with advanced age, independent of renal function (Fig. 3). Since advanced age is often associated with a reduction in renal function, the combined effect of both was assessed by simulating HbA1c lowering based on real patient data from the subset of patients aged 75-85 years [median eGFR 63 (95\% CI 21, 93) $\left.\mathrm{mL} / \mathrm{min} / 1.73 \mathrm{~m}^{2}\right]$, as well as in a subset of patients with renal impairment (study 10). The simulations indicated that for patients with an eGFR of $45-60 \mathrm{~mL} / \mathrm{min} / 1.73 \mathrm{~m}^{2}$, the empagliflozin 10- and 25-mg doses produced clinically meaningful reductions from baseline HbA1c values. This also holds true for the population of elderly patients (Table 3 ).

When comparing observed with simulated HbA1c changes from baseline, the observed change from baseline in the subgroups with moderate or severe renal impairment (eGFR $30-60$ and $45-60 \mathrm{~mL} / \mathrm{min} / 1.73 \mathrm{~m}^{2}$ ) should be interpreted with caution, in view of the small number of patients with moderate renal impairment who received 10-mg empagliflozin $(n=5)$ in the evaluated population. The simulations themselves were based on final parameter estimates from the overall FPG/ HbA1c dataset, where information from patients with chronic kidney disease (CKD) stage 3 ( $n=608$ patients) and stage $4(n=82$ patients) was available for identification of the influence of eGFR on FPG/HbA1c lowering, and 
hence, covariate influences could be reliably estimated. Overall, results from the simulations are in line with clinical findings, which showed that in patients with T2DM and stage 2 or 3 $\mathrm{CKD}$, the addition of empagliflozin as an add-on to existing antidiabetic therapy produced significant reductions in HbA1c. These clinical observations also included reductions in blood pressure and body weight in patients with stage 3 CKD [28]. However, for patients with severe renal impairment (stage 4 CKD), no reduction in HbA1c was observed [28].

For patients receiving background SU therapy, a greater reduction in FPG and HbA1c was demonstrated compared with a reference patient with no background antidiabetic therapy. Although this observation might be the result of a study effect (since most patients taking background SU therapy came from two studies: 6 and 9), data suggest that empagliflozin may improve $\beta$-cell function [29], and hence, might lead to a better response to pre-existing SU treatment. In contrast, concomitant metformin therapy did not show a significant influence on the effect of empagliflozin on FPG and HbA1c lowering. The finding of an increase in $k_{\mathrm{HbA1} \text {, out }}$ of $50.6 \%$ for patients on metformin is most probably related to a slightly reduced baseline HbA1c among patients receiving prior metformin treatment.

\section{CONCLUSIONS}

In conclusion, the population $\mathrm{PK}$ and $\mathrm{E}-\mathrm{R}$ models adequately described the PK, efficacy (reductions in FPG and HbA1c), and tolerability (hypoglycemia, genital infections, UTI, volume depletion) of empagliflozin. Variability in empagliflozin exposure was primarily affected by eGFR (increase in exposure less than twofold in patients with severe renal impairment).
Compared with placebo, empagliflozin therapy was associated with an exposure-independent increase in the incidence of genital infection and no significant change in the risk of UTI, hypoglycemia, or volume depletion. Consistent with the mode of action, the efficacy of empagliflozin was increased with elevated glucose levels and attenuated with decreasing renal function despite an increase in its exposure (less than twofold), but was still maintained to nearly half the maximal effect with eGFR as low as $30 \mathrm{~mL} / \mathrm{min} / 1.73 \mathrm{~m}^{2}$. All other investigated covariates including sex, BMI, race, and age did not alter the PK or efficacy of empagliflozin to a clinically relevant extent. Overall, no dose adjustment is required for empagliflozin in the patient population for which the drug is approved.

\section{ACKNOWLEDGMENTS}

All authors had full access to all of the data in this analysis, and take complete responsibility for the integrity of the data and accuracy of the data analysis. All named authors meet the International Committee of Medical Journal Editors (ICMJE) criteria for authorship for this manuscript, take responsibility for the integrity of the work as a whole, and have given final approval to the version to be published. All authors were fully responsible for all content and editorial decisions, and were involved at all stages of manuscript development, including reviewing and revising the manuscript for scientific content, and have approved the final version. In addition: Kyle T. Baron and Matthew Riggs developed the analysis plan, executed all analyses (including model development, model selection, and model summary), and interpreted the findings. Sreeraj Macha contributed to data collection, data selection, 
the analysis plan, and interpretation of the results. Valerie Nock contributed to the simulations and interpretation of the results. Uli C. Broedl contributed to the analysis concept, the statistical analysis plan and interpretation of the findings. Silke Retlich contributed to the analysis strategy, data selection, the analysis plan, and the interpretation of results. The results of this study were presented at the Annual Meeting and Exposition of the American Association of Pharmaceutical Scientists, San Antonio, TX, USA, November 10-14, 2013 (Poster number T3231). Sponsorship for this study and article processing charges were funded by Boehringer Ingelheim. Medical writing assistance, supported financially by Boehringer Ingelheim, was provided by Jennifer Edwards, MB BS, of Envision Scientific Solutions during the preparation of this manuscript.

Disclosures. Kyle T. Baron and Matthew Riggs are employees of Metrum Research Group LLC, and served as paid consultants to Boehringer Ingelheim. Uli C. Broedl, Valerie Nock, and Silke Retlich are all employees of Boehringer Ingelheim. Sreeraj Macha was an employee of Boehringer Ingelheim at the time of the study; he is now an employee of Merck \& Co.

Compliance with Ethics Guidelines. This article is based on the analysis of data from previously conducted studies, and does not involve any new studies of human or animal subjects performed by any of the authors.

Open Access. This article is distributed under the terms of the Creative Commons Attribution-NonCommercial 4.0 International License (http://creativecommons.org/licenses/ by-nc/4.0/), which permits any noncommercial use, distribution, and reproduction in any medium, provided you give appropriate credit to the original author(s) and the source, provide a link to the Creative Commons license, and indicate if changes were made.

\section{REFERENCES}

1. Bergenstal RM, Bailey CJ, Kendall DM. Type 2 diabetes: assessing the relative risks and benefits of glucose-lowering medications. Am J Med. 2010;123(374):e9-18.

2. Hernandez AV, Usmani A, Rajamanickam A, Moheet A. Thiazoldinediones and risk of heart failure in patients with or at high risk of type 2 diabetes mellitus: a meta-analysis and meta-regression analysis of placebo-controlled randomized clinical trials. Am J Cardiovasc Drugs. 2011;11:115-28.

3. Bailey CJ. Renal glucose reabsorption inhibitors to treat diabetes. Trends Pharmacol Sci. 2011;32:63-71.

4. Nair S, Wilding JP. Sodium glucose cotransporter 2 inhibitors as a new treatment for diabetes mellitus. J Clin Endocrinol Metab. 2010;95:34-42.

5. Rave K, Nosek L, Posner J, Heise T, Roggen K, van Hoogdalem EJ. Renal glucose excretion as a function of blood glucose concentration in subjects with type 2 diabetes-results of a hyperglycaemic glucose clamp study. Nephrol Dial Transplant. 2006;21:2166-71.

6. Ruhnau B, Faber OK, Borch-Johnsen K, Thorsteinsson B. Renal threshold for glucose in non-insulin-dependent diabetic patients. Diabetes Res Clin Pract. 1997;36:27-33.

7. Heise T, Seewaldt-Becker E, Macha S, et al. Safety, tolerability, pharmacokinetics and pharmacodynamics following 4 weeks' treatment with empagliflozin once daily in patients with type 2 diabetes. Diabetes Obes Metab. 2013;15:613-21.

8. Ferrannini E, Seman L, Seewaldt-Becker E, Hantel S, Pinnetti S, Woerle HJ. A Phase IIb, randomized, placebo-controlled study of the SGLT2 inhibitor empagliflozin in patients with type 2 diabetes. Diabetes Obes Metab. 2013;15:721-8.

9. Seman L, Macha S, Nehmiz G, et al. Empagliflozin (BI 10773), a potent and selective SGLT2 inhibitor, induces dose-dependent glucosuria in healthy subjects. Clin Pharm Drug Dev. 2013;2:152-61. 
10. Sarashina A, Koiwai K, Seman LJ, et al. Safety, tolerability, pharmacokinetics and pharmacodynamics of single doses of empagliflozin, a sodium glucose cotransporter 2 (SGLT2) inhibitor, in healthy Japanese subjects. Drug Metab Pharmacokinet. 2013;28:213-9.

11. Kanada S, Koiwai K, Taniguchi A, Sarashina A, Seman L, Woerle HJ. Pharmacokinetics, pharmacodynamics, safety and tolerability of 4 weeks' treatment with empagliflozin in Japanese patients with type 2 diabetes mellitus. J Diabetes Invest. 2013;4:613-7.

12. Heise T, Seman L, Macha S, et al. Safety, tolerability, pharmacokinetics, and pharmacodynamics of multiple rising doses of empagliflozin in patients with type 2 diabetes mellitus. Diabetes Ther. 2013;4:331-45.

13. Roden M, Weng J, Elibracht J, et al. Empagliflozin monotherapy with sitagliptin as an active comparator in patients with type 2 diabetes: a randomised, double-blind, placebo-controlled, phase 3 trial. Lancet Diabetes Endocrinol. 2013;1:208-19.

14. Häring HU, Merker L, Seewaldt-Becker E, et al. Empagliflozin as add-on to metformin in patients with type 2 diabetes: a 24-week, randomized, double-blind, placebo-controlled trial. Diabetes Care. 2014;37:1650-9.

15. Kovacs CS, Seshiah V, Swallow R, et al. Empagliflozin improves glycaemic and weight control as add-on therapy to pioglitazone or pioglitazone plus metformin in patients with type 2 diabetes: a 24-week, randomized, placebo-controlled trial. Diabetes Obes Metab. 2014;16:147-58.

16. Rosenstock J, Jelaska A, Frappin G, et al. Improved glucose control with weight loss, lower insulin doses, and no increased hypoglycemia with empagliflozin added to titrated multiple daily injections of insulin in obese inadequately controlled type 2 diabetes. Diabetes Care. 2014;37:1815-23.

17. Rosenstock J, Seman LJ, Jelaska A, et al. Efficacy and safety of empagliflozin, a sodium glucose cotransporter 2 (SGLT2) inhibitor, as add-on to metformin in type 2 diabetes with mild hyperglycaemia. Diabetes Obes Metab. 2013;15:1154-60.

18. Riggs MM, Seman LJ, Staab A, et al. Exposure-response modelling for empagliflozin, a sodium glucose cotransporter 2 (SGLT2) inhibitor, in patients with type 2 diabetes. $\mathrm{Br} \mathrm{J}$ Clin Pharmacol. 2014;78:1407-18.

19. Riggs MM, Staab A, Seman L, et al. Population pharmacokinetics of empagliflozin, a sodium glucose cotransporter 2 inhibitor, in patients with type 2 diabetes. J Clin Pharmacol. 2013;53:1028-38.

20. Burnham KP, Anderson DR. Model selection and multimodel inference: a practical information-theoretic approach. New York: Springer-Verlag; 2002.

21. Samtani MN. Simple pharmacometric tools for oral anti-diabetic drug development: competitive landscape for oral non-insulin therapies in type 2 diabetes. Biopharm Drug Dispos. 2010;31:162-77.

22. Gastonguay MR. Full covariate models as an alternative to methods relying on statistical significance for inferences about covariate effects: a review of methodology and 42 case studies. 2011. http://www.page-meeting.org/ default.asp?abstract=2229\#. Accessed 28 Apr 2016.

23. R Development Core Team. R: a language and environment for statistical computing. Vienna: $\mathrm{R}$ Foundation for Statistical Computing;2008.

24. Ferrannini E, Berk A, Hantel S, et al. Long-term safety and efficacy of empagliflozin, sitagliptin, and metformin: an active-controlled, parallel-group, randomized, 78-week open-label extension study in patients with type 2 diabetes. Diabetes Care. 2013;36:4015-21.

25. Sarashina A, Ueki K, Sasaki T, et al. Effect of renal impairment on the pharmacokinetics, pharmacodynamics, and safety of empagliflozin, a sodium glucose cotransporter 2 inhibitor, in Japanese patients with type 2 diabetes mellitus. Clin Ther. 2014;36:1606-15.

26. Macha S, Mattheus M, Halabi A, Pinnetti S, Woerle HJ, Broedl UC. Pharmacokinetics, pharmacodynamics and safety of empagliflozin, a sodium glucose cotransporter 2 (SGLT2) inhibitor, in subjects with renal impairment. Diabetes Obes Metab. 2014;16:215-22.

27. Scheen AJ. Pharmacodynamics, efficacy and safety of sodium-glucose co-transporter type 2 (SGLT2) inhibitors for the treatment of type 2 diabetes mellitus. Drugs. 2015;75:33-59.

28. Barnett AH, Mithal A, Manassie J, et al. Efficacy and safety of empagliflozin added to existing antidiabetes treatment in patients with type 2 diabetes and chronic kidney disease: a randomised, double-blind, placebo-controlled trial. Lancet Diabetes Endocrinol. 2014;2:369-84.

29. Ferrannini E, Muscelli E, Frascerra S, et al. Metabolic response to sodium-glucose cotransporter 2 inhibition in type 2 diabetic patients. J Clin Invest. 2014;124:499-508. 